\title{
Multiband Polarization Insensitive and Tunable Terahertz Metamaterial Perfect Absorber Based on The Heterogeneous Structure of Graphene
}

\author{
Amirhossein Norouzi Razani \\ Semnan University \\ Pejman Rezaei ( $\Delta$ prezaei@semnan.ac.ir) \\ Semnan University https://orcid.org/0000-0002-1266-3229
}

\section{Research Article}

Keywords: Metamaterial perfect absorber, Multi-band, Graphene, Tunable, Polarization insensitive, Terahertz

Posted Date: May 4th, 2021

DOI: https://doi.org/10.21203/rs.3.rs-438846/v1

License: (c) (i) This work is licensed under a Creative Commons Attribution 4.0 International License. Read Full License 


\title{
Multiband Polarization Insensitive and Tunable Terahertz Metamaterial Perfect Absorber Based on The Heterogeneous Structure of Graphene
}

\author{
Amirhossein Norouzi Razani, Pejman Rezaei* \\ * Corresponding Author Email: prezaei@semnan.ac.ir
}

\begin{abstract}
In this paper, we present and investigate a multi-band metamaterial perfect absorber (MPA) based on the heterogeneous structure of graphene with $\mathrm{Cu}$ and $\mathrm{SiO}_{2}$ substrates. The top layer of structure consist of one graphene disk at the center and four graphene solid triangle with semicircular cuts on them that surround the central disk. This heterogeneous structure causes us to achieve $97.06 \%, 94.71 \%, 99.7 \%$ and $99.5 \%$ perfect absorptions peaks at $28239.7 \mathrm{~nm}, 31048.9 \mathrm{~nm}, 50898.6 \mathrm{~nm}$ and $70689.1 \mathrm{~nm}$, respectively. The absorption mechanism based on electric fields has been investigated. We can shift the wavelength of absorption peaks to our required wavelength by changing the Fermi level $(\mu \mathrm{c})$ of graphene. Two absorption peaks of this absorber remain unchanged in different incident angle. In addition, very important point about this structure is that it is not sensitive to polarization and this feature makes the proposed absorber very suitable for applications such as imaging, filtering, sensing and detecting applications.
\end{abstract}

Key word: Metamaterial perfect absorber, Multi-band, Graphene, Tunable, Polarization insensitive, Terahertz.

\section{Introduction}

With the increasing development of communication technology and the use of frequency in the terahertz range, the need for tools and equipment in this frequency range is inevitable. This need drew the attention of all terahertz range researchers to metamaterials.

A metamaterial is any material engineered to have a property that is not found in naturally occurring materials [1]. Metamaterials have special properties such as negative refraction $[2,3]$, cloak [4, 5] superlunary [6, 7] and backward wave [8,9], and for this reason, they have been highly regarded by researchers in the field of electromagnetism.

One of the most important and practical metamaterials is graphene. Graphene is a metamaterial made of graphite, and graphite itself is made up of layers of carbon atoms arranged hexagons. Each of these layers is fastened together with a strong bond. By breaking these bonds according to Fig. 1, we can reach the graphene metamaterial, which is a single layer of carbon atoms, and because of this, it is called a twodimensional metamaterial [10].

Graphene has received a great deal of attention in the construction of antennas and alternating structures, including absorbers, electromagnetic shields, and electromagnetic sensors, due to its small size, two-dimensionality, high conductivity and its adjustable conductivity $[11,12]$. In the last decade, we have done research and development in the field of design and improvement of graphene structures.
Due to the use of graphene in antennas has brought us significant benefit such as extreme miniaturization, monolithic integration with graphene RF Nano- electronics, efficient dynamic tuning, and even transparency and mechanical flexibility [13], the idea of designing graphene antennas has been a step to ahead.

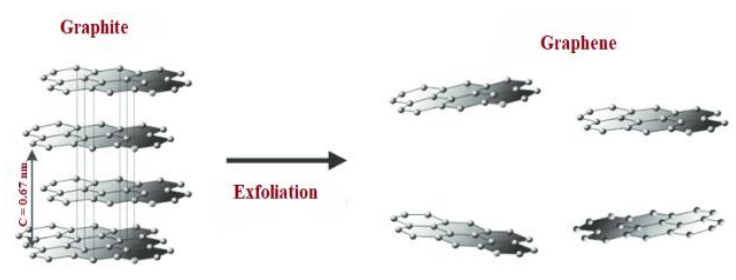

Fig. 1 Exfoliation of graphite to create graphene

Since we can change the chemical potential of graphene by external bias voltage or chemical doping, graphene is an adjustable metamaterial [14]. We used this graphene capability to design the antennas with performance in far-field and terahertz range central frequency due to control its polarization just by changing the Fermi energy level of the graphene [15-18].

Another application of graphene is the design and construction of absorbers that operate in the microwave [19, 20] and terahertz [21, 22] range. Electromagnetic absorbers are specifically selected or designed materials that can inhibit the reflection or transmission of electromagnetic radiation. Features that are important in absorbers are full absorption (close to $100 \%$ adsorption), insensitivity to polarization and incident angle of radiation. 
Absorbers that use metamaterials in their design and have absorption close to $100 \%$ are called metamaterial perfect absorbers (MPA) [23-26]. There are two types of absorbers: resonant absorbers [27-29] and wideband absorbers [30-32]. Terahertz band absorbers have many applications such as sensing, imaging, modulating, detection, filtering, etc. [33-35].

The mechanism of absorption of multilayer absorbers is such that the underlying conductive layer does not allow radiation to pass through the structure. In addition, the intermediate dielectric layer and the metamaterial placed on the dielectric must also be designed so that the waves reflected from inside the structure Have a Phase difference Aiming to neutralize each other, in order to the structure reach to the perfect absorption.

One of the characteristics that makes the absorber structure unique for different applications is the insensitivity of the structure to polarization [36] and incident angle [37].

In this paper, we present a multi-band perfect metamaterial absorber that has one graphene disk at the center and four graphene solid triangle with semicircular cuts on them that surround the central disk. The dielectric of the middle layer and the conductive of underlying layer of the structure are $\mathrm{SiO}_{2}$ and $\mathrm{Cu}$, respectively. One of the advantages of this structure is in its construction, because in this structure, copper conductor is used in the lower layer to prevent radiation transmission, which is more economical than structures in which gold is used in the lower layer [38-41]. The proposed absorber is a resonant absorber that has four perfect absorption peaks, which are $97.06 \%, 94.71 \%, 99.7 \%$ and $99.5 \%$ at wavelengths of $28239.7 \mathrm{~nm}, 31048.9 \mathrm{~nm}, 50898.6$ $\mathrm{nm}$ and $70689.1 \mathrm{~nm}$, respectively.

\section{Structure and Design}

As shown in Fig. 2(a), we present a three-layer absorber. The bottom layer of the absorber is a cube with dimensions $\mathrm{L} \times \mathrm{L}(\mathrm{L}=3 \mu \mathrm{m})$ and a thickness of $0.5 \mu \mathrm{m}$. The material of this substrate is copper with conductivity of $\sigma_{\mathrm{Cu}}=5.813 \times 10^{7} \mathrm{~S} / \mathrm{m}$ [42]. This conductor has the task of reducing the radiation transmission from the structure to zero. Our middle layer is a cube with the same dimensions $\mathrm{L} \times \mathrm{L}$ and a thickness of $4.9 \mu \mathrm{m}$. In addition, its dielectric material is $\mathrm{SiO}_{2}$ with a relative permittivity of $\epsilon_{\mathrm{r}}=$ $3.9[43,44]$.

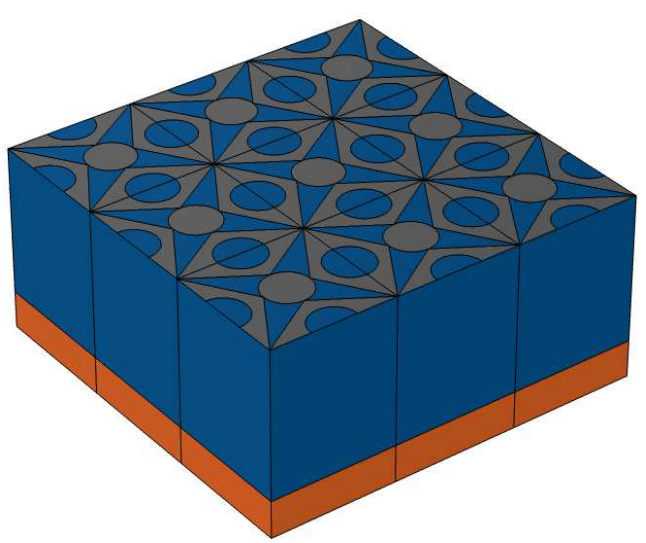

(a)

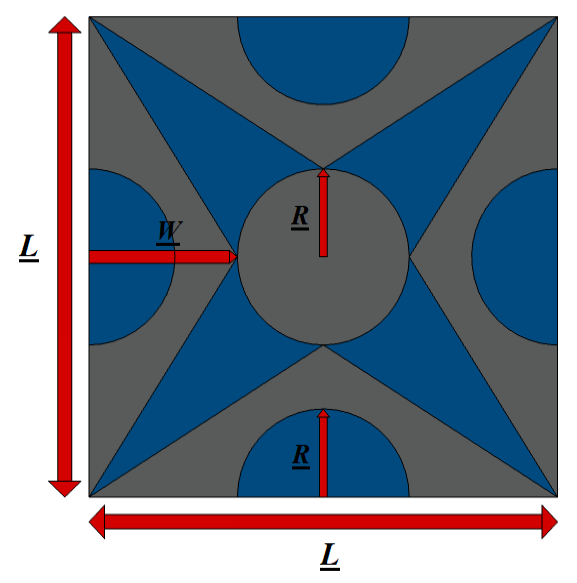

(b)

Fig. 2 (a) Unit cells structure from three-dimensional view. (b) Dimensions of the absorber structure from a two-dimensional view. (These figures were prepared using COMSOL Multiphysics software).

As mentioned, graphene is a two-dimensional material that is placed on our $\mathrm{SiO}_{2}$ layer. According to Fig. 2 (b), the central graphene disk has a radius of $\mathrm{R}=0.55 \mu \mathrm{m}$. The four triangles that surround the central disk are equal in size. The height of each triangle is $\mathrm{w}=0.95 \mu \mathrm{m}$ and the base of each of them is equal to $\mathrm{L}=3 \mu \mathrm{m}$. We also cut each triangle into a semicircle so that all the semicircles are equal and have a radius $\mathrm{R}=0.55 \mu \mathrm{m}$.

\section{Simulation Results}

We performed the simulations at room temperature ( $\mathrm{T}=300 \mathrm{~K}$ ) using the CST Studio Suite software.

As shown in Fig. 3, by setting the parameters of graphene to $\mu_{\mathrm{c}}=0.8 \mathrm{eV}$ and $\tau=1.6 \mathrm{ps}$, the structure has four perfect absorption peaks, which are $97.06 \%$, $94.71 \%, 99.7 \%$ and $99.5 \%$ at $28239.7 \mathrm{~nm}, 31048.9$ $\mathrm{nm}, 50898.6 \mathrm{~nm}$ and $70689.1 \mathrm{~nm}$, respectively. 


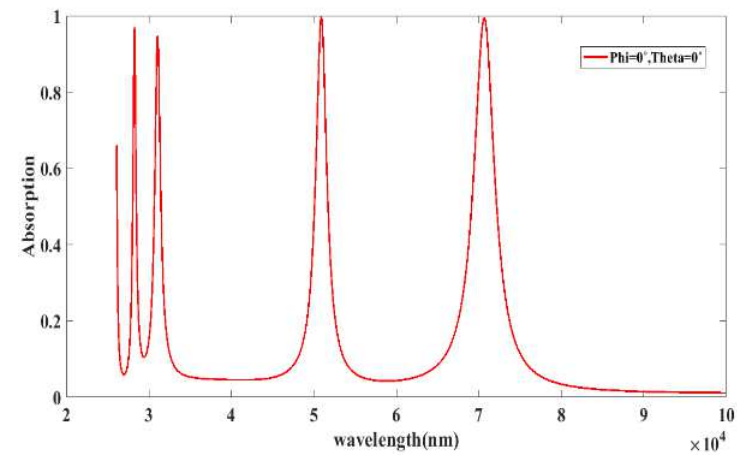

Fig. 3 Absorption spectra of structure with $\mu \mathrm{c}=0.8 \mathrm{eV}$ and $\tau=1.6 \mathrm{ps}$.

This structure is tunable; it means that we can shift the frequency of absorption to a required frequency for the intended application or increase and decrease the amount of absorption at the desired frequency by changing Fermi level or relaxation time of graphene. We changed the Fermi level of graphene to different values, and you can see the frequency changes of the absorption peaks in Fig. 4(a). We also changed the relaxation time of graphene in Fig. 4(b), where you can see the changes in the absorption peaks of the structure.

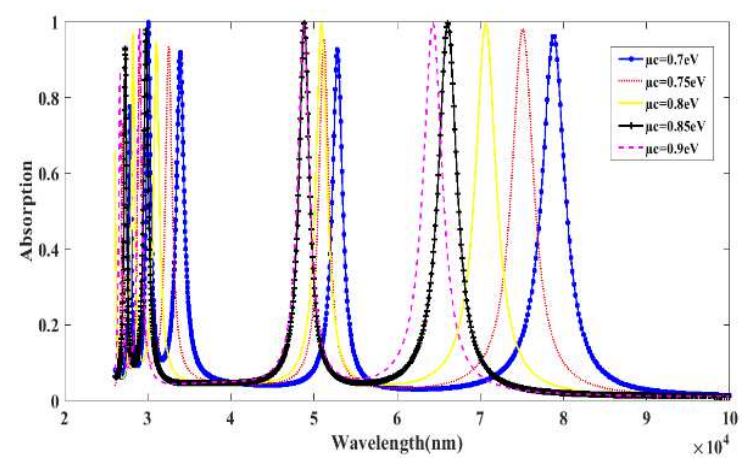

(a)

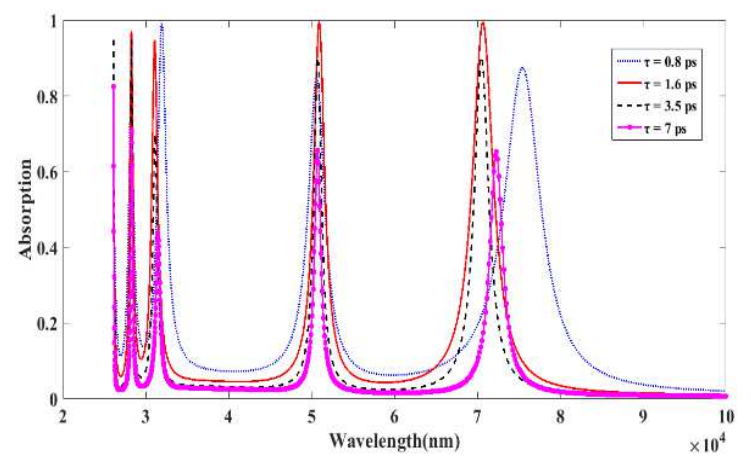

(b)

Fig. 4 Absorption spectra of structure for (a) different Fermi level $\mu_{\mathrm{c}}$ at $\tau=1.6 \mathrm{ps}$ and (b) different relaxation time $\tau$ at $\mu_{\mathrm{c}}=0.8 \mathrm{eV}$.
As you can see in Fig. 5, one of the good advantages of this structure is that we can increase the number of absorption peaks by changing the Fermi level of graphene (for example changing it to $0.5 \mathrm{eV}$ ) and changing the relaxation time of graphene (for example changing it to $0.5 \mathrm{ps}$ ). By this setting our structure has six absorption peaks, four of those are above $90 \%$, one of those is above $85 \%$ and the other one is above $75 \%$. This setting is used for applications where the number of absorption peaks takes precedence over the absorption rate.

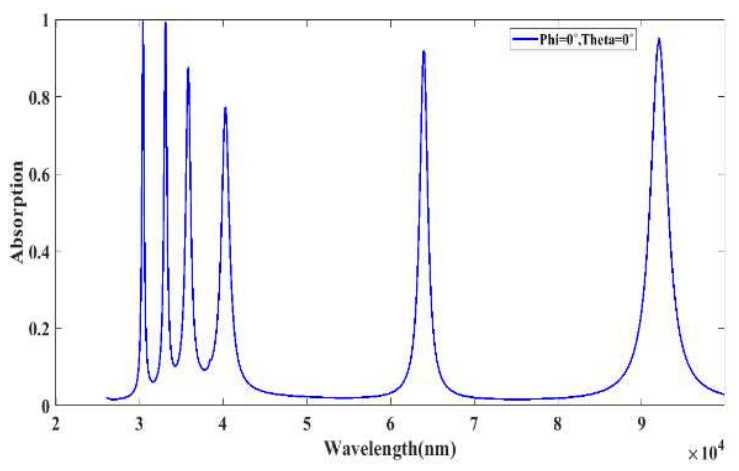

Fig. 5 Increasing the number of absorption peaks by setting $\mu_{\mathrm{c}}=0.5 \mathrm{eV}$ and $\tau=3 \mathrm{ps}$.

One feature that is very important in absorbers is the insensitivity of the structure to polarization. As you can see in Fig. 6(a), the absorber presented in this paper is polarization insensitive because the absorption peaks remain unchanged at different angles of polarization. This feature makes the proposed absorber very suitable for applications such as imaging, detecting, filtering, sensing, etc.

As shown in Fig. 6(b), it is obtained that the absorption peaks at wavelengths $28239.7 \mathrm{~nm}$ and $50898.5 \mathrm{~nm}$ with different incident angles (From 0 to 45 degrees) remain almost unchanged. Especially at the $28239.7 \mathrm{~nm}$ peak, the absorption rate increases slightly with increasing incident angle. This makes the proposed absorber more widely used in various applications.

\section{Discussion and Comparison}

The absorption mechanism of this absorber is such that the bottom layer of copper does not allow radiation to pass through the structure. In addition, the middle layer of $\mathrm{SiO}_{2}$ and graphene placed on the layer of $\mathrm{SiO}_{2}$ is also designed in such a way that the waves reflected from inside the structure have a phase difference and neutralize each other, thus the structure reaches four full absorption peaks. 


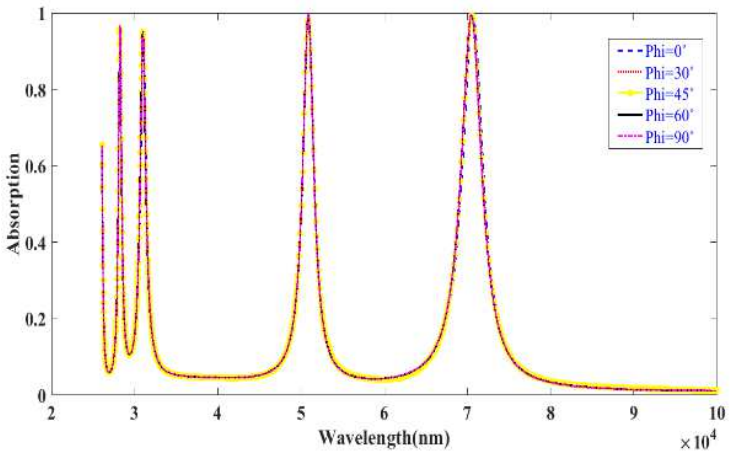

(a)

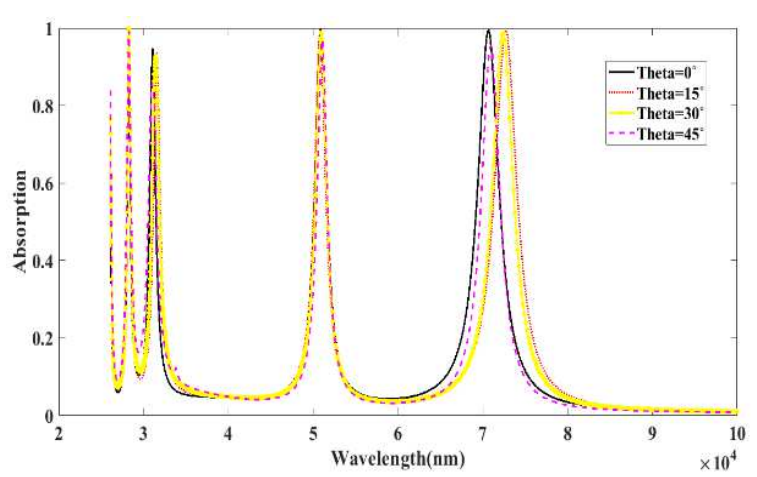

(b)

Fig. 6 Absorption spectra of structure for (a) different polarization angles and (b) different incident angles.

(a)

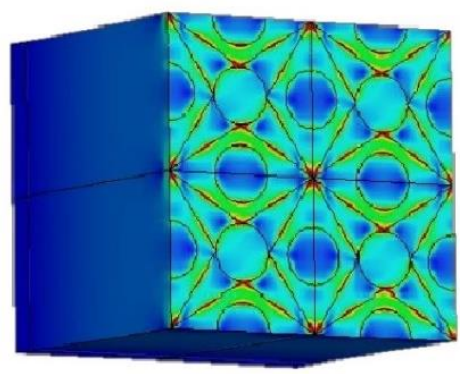

(c)

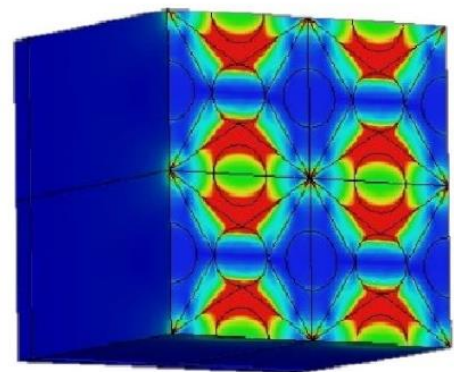

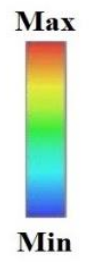

(b)
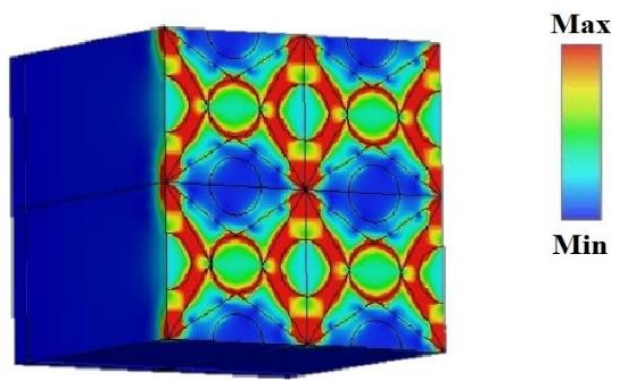

$\operatorname{Max}$
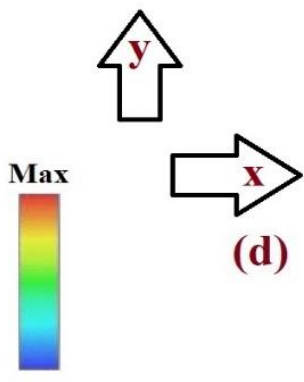

(d)

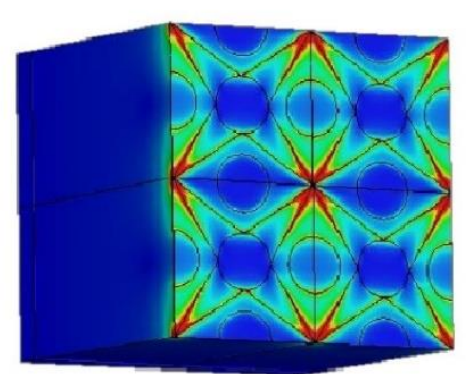

Max

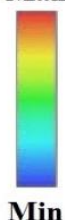

Fig. 7 Electric field [real (Ez)] distributions in the normal incidence of TE waves at wavelengths of (a) $\lambda=28239.7 \mathrm{~nm}$ (b) $\lambda=31048.9 \mathrm{~nm}$ (c) $\lambda=50898.6 \mathrm{~nm}$ (d) $\lambda=70689.1 \mathrm{~nm}$

Fig. 7 shows the electric field [real (Ez)] distributions in the normal incidence of TE waves at wavelengths of absorption peaks. As you can see in Fig. 7 (a), the field distribution is mainly concentrated on the intersection of the graphene disk and the graphene triangle located in the y direction, Perimeter of semicircular cuts located in the $y$ direction, the bottom two corners and along the sides of each graphene triangle. Therefore, we can conclude that the first resonance at wavelength of $\lambda=28239.7 \mathrm{~nm}$ is due to resonance at these points. Fig. 7 (b) shows that the electric field is mainly concentrated on the surface of the graphene triangles located in the $\mathrm{x}$ direction and on the inner edges of the graphene disk.
As a result, the second resonance at wavelength of $\lambda=31048.9 \mathrm{~nm}$ can be considered due to the graphene resonance at these points. A similar analysis of Fig. 7 (c) shows that the electric field is concentrated on a part of the surface of graphene triangles located in the $y$ direction along the semicircular cuts and the graphene disk, and this concentration extends to the edges of the graphene disk. So the third resonance at the wavelength of $\lambda=50898.6 \mathrm{~nm}$ is attributed to the resonance of these points. Finally, in Fig. 7 (d), it is clear that the electric field is concentrated in the lower two corners and along the sides of the triangles located in the $\mathrm{x}$ direction. Therefore, we can conclude that the fourth resonance at wavelength of $\lambda=70689.1 \mathrm{~nm}$ is due to resonance at these points. 
Table 1 Comparison between the features of the absorber structure of different articles with the features of the proposed absorber structure in this paper

\begin{tabular}{|c|c|c|c|c|}
\hline References & $\begin{array}{l}\text { Absorption } \\
\text { freq. / wavelengths } \\
\text { (THz/nm) }\end{array}$ & $\begin{array}{l}\text { Absorption } \\
\text { peaks }\end{array}$ & $\begin{array}{l}\text { Polarization } \\
\text { sensitivity }\end{array}$ & Incident angle sensitivity \\
\hline [45] & $\begin{array}{l}0.60 \mathrm{THz} \\
0.80 \mathrm{THz} \\
1.74 \mathrm{THz} \\
2.33 \mathrm{THz} \\
2.75 \mathrm{THz} \\
3.63 \mathrm{THz} \\
\end{array}$ & $\begin{array}{l}99.10 \% \\
99.90 \% \\
98.77 \% \\
98.82 \% \\
99.99 \% \\
99.63 \% \\
\end{array}$ & $\begin{array}{l}\text { Sensitive to } \\
\text { polarization }\end{array}$ & No analysis has been done \\
\hline [46] & $\begin{array}{l}4.95 \mathrm{THz} \\
9.2 \mathrm{THz}\end{array}$ & $\begin{array}{l}99.8 \% \\
99.6 \%\end{array}$ & $\begin{array}{l}\text { No analysis has } \\
\text { been done }\end{array}$ & No analysis has been done \\
\hline [47] & $\begin{array}{c}1.054 \mathrm{THz} \\
2.16 \mathrm{THz} \\
3.59 \mathrm{THz} \\
3.87 \mathrm{THz}\end{array}$ & $\begin{array}{c}90.6 \% \\
97.2 \% \\
93.95 \% \\
99.61 \%\end{array}$ & $\begin{array}{c}\text { Sensitive to } \\
\text { polarization at } \\
\text { three absorption } \\
\text { peaks }\end{array}$ & $\begin{array}{l}\text { Insensitive to incident angle } \\
\text { (at a wide angle) }\end{array}$ \\
\hline [48] & $\begin{array}{c}0.8 \mathrm{THz} \\
1.72 \mathrm{THz} \\
3.38 \mathrm{THz}\end{array}$ & $\begin{array}{l}99.43 \% \\
99.92 \% \\
99.58 \%\end{array}$ & $\begin{array}{l}\text { Polarization } \\
\text { insensitive }\end{array}$ & $\begin{array}{c}\text { Insensitive to incident angle } \\
\text { (at a wide angle) } \\
\text { at two absorption peaks }\end{array}$ \\
\hline [49] & $0.245 \mathrm{THz}$ & $97.5 \%$ & $\begin{array}{l}\text { Polarization } \\
\text { insensitive }\end{array}$ & $\begin{array}{l}\text { Insensitive to incident angle } \\
\text { (at a wide angle) }\end{array}$ \\
\hline$[50]$ & $\begin{array}{l}0.114 \mathrm{THz} \\
0.181 \mathrm{THz} \\
\end{array}$ & $\begin{array}{l}97.9 \% \\
99.8 \% \\
\end{array}$ & $\begin{array}{c}\text { Polarization } \\
\text { insensitive }\end{array}$ & $\begin{array}{l}\text { Insensitive to incident angle } \\
\text { (at a wide angle) }\end{array}$ \\
\hline$[51]$ & $\begin{array}{l}1.16 \mathrm{THz} \\
2.73 \mathrm{THz} \\
4.57 \mathrm{THz}\end{array}$ & $\begin{array}{l}99.6 \% \\
99.8 \% \\
99.5 \%\end{array}$ & $\begin{array}{l}\text { Polarization } \\
\text { insensitive }\end{array}$ & $\begin{array}{c}\text { Insensitive to incident angle } \\
\text { (at a wide angle) at two } \\
\text { absorption peaks }\end{array}$ \\
\hline$[52]$ & $\begin{array}{l}0.80 \mathrm{THz} \\
2.89 \mathrm{THz} \\
3.98 \mathrm{THz} \\
4.36 \mathrm{THz}\end{array}$ & $\begin{array}{l}\text { In an average of } \\
97.54 \%\end{array}$ & $\begin{array}{l}\text { Polarization } \\
\text { insensitive }\end{array}$ & No analysis has been done \\
\hline [53] & $\begin{array}{l}1.92 \mathrm{THz} \\
4.62 \mathrm{THz} \\
8.81 \mathrm{THz} \\
\end{array}$ & $\begin{array}{l}98.4 \% \\
98.3 \% \\
99.6 \% \\
\end{array}$ & $\begin{array}{l}\text { No analysis has } \\
\text { been done }\end{array}$ & No analysis has been done \\
\hline [54] & $\begin{array}{c}2.59 \mathrm{THz}(\mathrm{OS}) \\
3.03 \mathrm{THz}(\mathrm{OS}) \\
6.54-7.10 \mathrm{THz}(\mathrm{OS}) \\
3.12 \mathrm{THz}(\mathrm{TS}) \\
6.00-7.21 \mathrm{THz}(\mathrm{TS})\end{array}$ & Larger than $90 \%$ & $\begin{array}{l}\text { Polarization } \\
\text { insensitive in both } \\
\text { TS and OS }\end{array}$ & $\begin{array}{l}\text { Sensitive to incident angle } \\
\text { in both TS and OS }\end{array}$ \\
\hline$[55]$ & $\begin{array}{c}1085.03 \mathrm{~nm} \\
1131.48 \mathrm{~nm} \\
1187 \mathrm{~nm} \\
1365.35 \mathrm{~nm}\end{array}$ & $\begin{array}{l}95.88 \% \\
99.81 \% \\
97.44 \% \\
95.30 \%\end{array}$ & $\begin{array}{l}\text { Insensitive to } \\
\text { polarization in } \\
\text { TM mode }\end{array}$ & $\begin{array}{c}\text { Insensitive to incident angle } \\
\text { (at a wide angle) } \\
\text { in TM mode }\end{array}$ \\
\hline $\begin{array}{l}\text { Proposed } \\
\text { absorber }\end{array}$ & $\begin{array}{l}28239.7 \mathrm{~nm} \\
31048.9 \mathrm{~nm} \\
50898.6 \mathrm{~nm} \\
70689.1 \mathrm{~nm}\end{array}$ & $\begin{array}{c}97.06 \% \\
94.71 \% \\
99.7 \% \\
99.5 \%\end{array}$ & $\begin{array}{l}\text { Polarization } \\
\text { insensitive }\end{array}$ & $\begin{array}{c}\text { Insensitive to incident angle } \\
\text { (at a wide angle) } \\
\text { at two absorption peaks }\end{array}$ \\
\hline
\end{tabular}


As mentioned, more absorption peaks, high absorption rate, insensitivity to polarization and incident angle of incident wave are some of the features that distinguish the metamaterial absorber. For this purpose, in Table 1, we have compared the metamaterial absorber presented in this paper with some of the metamaterial absorbers presented in recent years in terms of having or not having these properties.

As it is known, the proposed absorber has more absorption peaks than some of the compared absorbers and has a higher absorption rate than some of them. In addition, the proposed absorber in comparison with some of the absorbers presented in Table 1 has the advantage of insensitivity to polarization and the incident angle at its two absorption peaks. As a result, the proposed absorber is more flexible and suitable for many applications such as imaging, detecting, sensing, filtering, etc. due to multi-band absorption, complete absorption, insensitivity to polarization and insensitivity to the incident angle at its two absorption peaks.

\section{Conclusion}

In recent years, many terahertz absorbers have been introduced. The absorber presented in this paper is a multi-band metamaterial perfect absorber that has the ability to adjust and increase the absorption peaks only by changing the Fermi level or the graphene relaxation time, without the need for structural change. In addition, this absorber is insensitive to polarization and its two absorption peaks remain almost unchanged in various incident angle (from 0 to 45 degrees). All these features make the absorber very suitable for applications such as imaging, detection, sensing and filtering.

\section{Acknowledgments}

This research was supported by Semnan University.

\section{Funding}

No funding was received for this research.

\section{Authors' Contributions}

Amirhossein Norouzi Razani: conceptualization, methodology, software, result analysis, writing - original draft, writing - review and editing. Pejman Rezaei: validation, data curation, writingreview and editing, supervision.

\section{Data Availability}

All data generated or analyzed during this study are included in this published article.

\section{Compliance with Ethical Standards}

Competing Interests The authors declare that they have no conflict of interest.
Ethical Approval We declare that this article is original, has not been published before, and is not currently considered for publication elsewhere. We confirm that the manuscript has been read and approved by all named authors and that there are no other persons who satisfied the criteria for authorship but are not listed. We further confirm that the order of authors listed in the manuscript has been approved by all of us.

Consent for Publication Written informed consent for publication was obtained from all participants.

Consent to Participate Written informed consent for participate was obtained from all participants.

\section{References}

1. Kshetrimayum RS (2004) A Brief Intro to Metamaterials. IEEE Potentials 23(5): 44-46

2. Veselago VG (1968) The electrodynamics of substances with simultaneously negative values of $\varepsilon$ and $\mu$. Soviet Phys Uspekhi 10(4):509-514

3. Valentine J, Zhang S, Zentgraf T, Ulin-Avila E, Genov DA, Bartal G, Zhang X (2008) Three-dimensional optical metamaterial with a negative refractive index. Nature 455(7211): 376-379

4. Cai W, Chettiar UK, Kildishev AV, Shalaev VM (2007) Optical cloaking with metamaterials. Nature Photonics 1(4):224-227

5. Shin D, Urzhumov Y, Jung Y, Kang G, Baek S, Choi M, Park H, Kim K, Smith DR (2012) Broadband electromagnetic cloaking with smart metamaterials. Nature Commun 3(1213):1-8

6. Pendry JB (2000) Negative refraction makes a perfect lens. Phys Rev Lett 85(18):3966-3969

7. Smith DR, Pendry JB, Wiltshire MCK (2004) Metamaterials and negative refractive index. Science 305(5685):788-792

8. Simovski CR, Belov PA, He SL (2003) Backward wave region and negative material parameters of a structure formed by lattices of wires and split-ring resonators. IEEE Trans Antennas Propag. 51(10):2582-2591

9. Song W, Teng Y, Zhang ZQ, Li JW, Sun J, Chen CH, Zhang LJ (2012) Rapid startup in relativistic backward wave oscillator by injecting external backward signal. Phys. Plasmas 19(083105): $1-4$

10. Katsnelson MI (2007) Graphene: carbon in two dimensions. Materials Today 10(1):20-27

11. Pop E, Varshney V, Roy AK (2012) Thermal properties of graphene: Fundamentals and applications. MRS Bulletin 37(12): 1273-1281

12. Ghods MM, Rezaei P (2018) Graphene-based Fabry-Perot resonator for chemical sensing applications at mid-infrared frequencies. IEEE Photon Technol Lett 30(22): 1917-1920

13. Perruisseau-Carrier J (2012) Graphene for antenna applications: Opportunities and challenges from microwaves to THz. Loughborough Antennas Propag Conf (LAPC), UK P. 1-4 14. Zamzam P, Rezaei P, Khatami SA (2021) Quad-band polarization-insensitive metamaterial perfect absorber based on bilayer graphene metasurface. Phys E: Low-dimension Systems Nanost 128:114621

15. Jafari Chashmi M, Rezaei P, Kiani N (2019) Reconfigurable graphene-based V-shaped dipole antenna: From quasi-isotropic to directional radiation pattern. Optik 184:421-427

16. Jafari Chashmi M., Rezaei P, Kiani N (2020) Polarization controlling of multi resonant graphene-based microstrip antenna. Plasmonics 15:417-426 
17. Kiani N, Tavakkol Hamedani F, Rezaei P (2021) Polarization controlling idea in graphene-based patch antenna. Optik 239: 166795

18. Jafari Chashmi M, Rezaei P, Kiani N (2020) Y-shaped graphene-based antenna with switchable circular polarization. Optik 200:163321

19. Grande M, Bianco GV, Perna FM, Capriati V, Capezzuto P, Scalora M, Bruno G, D’Orazio A (2019) Reconfigurable and optically transparent microwave absorbers based on deep eutectic solvent-gated graphene. Scientific Reports 9:5463

20. Ghods MM, Rezaei P (2018) Ultra-wideband microwave absorber based on uncharged graphene layers. Electromag Wav Appl 32(15):1950-1960

21. Wang Y, Song M, Pu M, Gu Y, Hu C, Zhao Z, Wang C, Yu H, Luo X (2016) Staked graphene for tunable terahertz absorber with customized bandwidth. Plasmonics 11:1201-1206

22. Gong J, Shi X, Lu Y, Hu F, Zong R, Li G (2021) Dynamically tunable triple-band terahertz perfect absorber based on graphene metasurface. Superlattices Microst 150:106797

23. Yao G, Ling F, Yue J, Luo C, Ji J, Yao J (2016) Dual-band tunable perfect metamaterial absorber in the $\mathrm{THz}$ range. Opt Express 24(2):1518-1527

24. Tran CM, Van Pham H, Nguyen HT, et al. (2019) Creating multiband and broadband metamaterial absorber by multiporous square layer structure. Plasmonics 14:1587-1592

25. Ke R, Liu W, Tian J, Yang R, Pei W (2020) Dual-band tunable perfect absorber based on monolayer graphene pattern. Results in Physics 18:103306

26. Zamzam P, Rezaei P (2021) A terahertz dual-band metamaterial perfect absorber based on metal-dielectric-metal multi-layer columns. Opt Quant Electron 53(2):109

27. Huang M, Cheng Y, Cheng Z, Chen H, Mao X, Gong R (2018) Based on graphene tunable dual-band terahertz metamaterial absorber with wide-angle. Optics Commun 415:194-201

28. Xu KD, Li J, Zhang A, Chen Q (2020) Tunable multi-band terahertz absorber using a single-layer square graphene ring structure with T-shaped graphene strips. Opt Express 28(8): 11482-11492

29. Feng Y, Hu ZD, Balmakou A, Khakhomov S, Semchenko I, Wang J, Liu D, Sang T (2020) Perfect narrowband absorber based on patterned graphene-silica multilayer hyperbolic metamaterials. Plasmonics 15:1869-1874

30. Deng Yw, Peng L, Liao X, Jiang X (2019) An ultra-broadband terahertz absorber based on coplanar graphene and gold hybridized metasurface. Plasmonics 14:1057-1061

31. Zhou R, Jiang T, Peng Z, Li Z, Zhang M, Wang S, Li L, Liang H, Ruan S, Su H (2021) Tunable broadband terahertz absorber based on graphene metamaterials and VO2. Optical Materials 114:110915

32. Li X, Feng G, Lin S (2021) Ultra-wideband terahertz absorber based on graphene modulation. Appl Opt 60(11):3170-3175

33. Meng T, Hu D, Zhu Q (2018) Design of a five-band terahertz perfect metamaterial absorber using two resonators. Opt Commun 415:151-155

34. Song Z, Wang K, Li J, Liu QH (2018) Broadband tunable terahertz absorber based on vanadium dioxide metamaterials. Opt Express 26(6):7148-7154

35. Bai, J, Shen W, Shi J, Xu W, Zhang S, Chang S (2021) A nonvolatile tunable terahertz metamaterial absorber using graphene floating gate. Micromachines 12(3):333

36. Verma VK, Mishra SK, Kaushal KK et al (2020) An octaband polarization insensitive terahertz metamaterial absorber using orthogonal elliptical ring resonators. Plasmonics 15:75-81

37. Pan M, Huang H, Fan B, Chen W, Li S, Xie Q, Xu F, Wei D, Fang J, (2021) Theoretical design of a triple-band perfect metamaterial absorber based on graphene with wide-angle insensitivity. Results in Physics 23:104037

38. Zhang J, Tian J, Li L, (2018) A dual-band tunable metamaterial near-unity absorber composed of periodic cross and disk graphene arrays. IEEE Photon J 10(2):1-12

39. Yi Z, Lin H, Niu G, Chen X, Zhou Z, Ye X, Duan T, Yi Y, Tang Y, Yi Y (2019) Graphene-based tunable triple-band plasmonic perfect metamaterial absorber with good anglepolarization-tolerance. Results in Physics 13:102149

40. Wu J (2019) Tunable multi-band terahertz absorber based on graphene nano-ribbon metamaterial. Phys Lett A 383:2589-2593

41. Feng S, Zhao Y, Liao YL (2020) Dual-band dielectric metamaterial absorber and sensing applications. Results Phys 18:103272

42. Tahseen MM, Kishk AA (2016) Wideband textile-based conformal antennas for WLAN band using conductive thread. 10th Europ Conf Antennas Propag, Davos, Switzerland 1-5

43. Chen PY, Alù A (2011) Atomically thin surface cloak using graphene monolayers. ACS Nano 5(7): 5855-5863

44. Deng XH, Liu JT, Yuan J, Wang TB, Liu NH (2014) Tunable $\mathrm{THz}$ absorption in graphene-based heterostructures. Opt. Express 22(24):30177-30183

45. Wang BX, Wang GZ, Sang T, Wang LL (2017) Six-band terahertz metamaterial absorber based on the combination of multiple-order responses of metallic patches in a dual-layer stacked resonance structure. Scientific Reports 7:41373

46. Xing R, Jian S (2018) A dual-band THz absorber based on graphene sheet and ribbons. Opt Laser Technol 100:129-132

47. Appasani B, Prince P, Ranjan RK, Gupta N, Verma VK (2019) A simple multi-band metamaterial absorber with combined polarization sensitive and polarization insensitive characteristics for terahertz applications. Plasmonics 14:737-742 48. Meng W, Que L, Lv J, Zhang L, Zhou Y, Jiang Y (2019) A triple-band terahertz metamaterial absorber based on buck Dirac semimetals. Results Phys 14:102461

49. Wang J, Lang T, Hong Z, Shen T, Wang G (2020) Tunable terahertz metamaterial absorber based on electricity and light modulation modes. Opt Mater Express 10(9):2262-2273

50. Li W, Cheng Y (2020) Dual-band tunable terahertz perfect metamaterial absorber based on strontium titanate (STO) resonator structure. Opt Commun 462:125265

51. Aksimsek S (2020) Design of an ultra-thin, multiband, microslot based terahertz metamaterial absorber. Electromag Wav Appl 34(16):2181-2193

52. Lou P, Wang BX, He Y, et al. (2020) Simplified design of quad-band terahertz absorber based on periodic closed-ring resonator. Plasmonics 15:1645-1651

53. Rahmanshahi M, Noori Kourani S, Golmohammadi S, Baghban H, Vahed H (2021) A tunable perfect THz metamaterial absorber with three absorption peaks based on nonstructured graphene. Plasmonics doi: 10.1007/s11468-021-01432-7

54. Wang ZL, Hu CX, Liu HB, Zhang HF (2021) A newfangled terahertz absorber tuned temper by temperature field doped by the liquid metal. Plasmonics 16:425-434

55. Jiang L, Yuan C, Li Z, Su J, Yi Z, Yao W, Wu P, Liu Z, Cheng S, Pan M (2021) Multi-band and high-sensitivity perfect absorber based on monolayer graphene metamaterial. Diamond Related Materials 111:108227 
Figures

\section{Graphite}

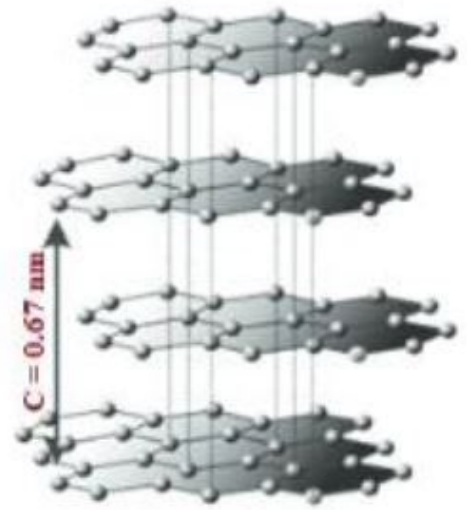

Figure 1

Exfoliation of graphite to create

\section{Graphene}
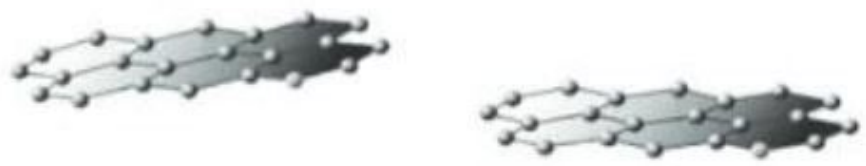

\section{Exfoliation}

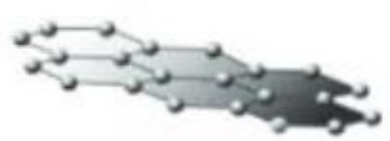




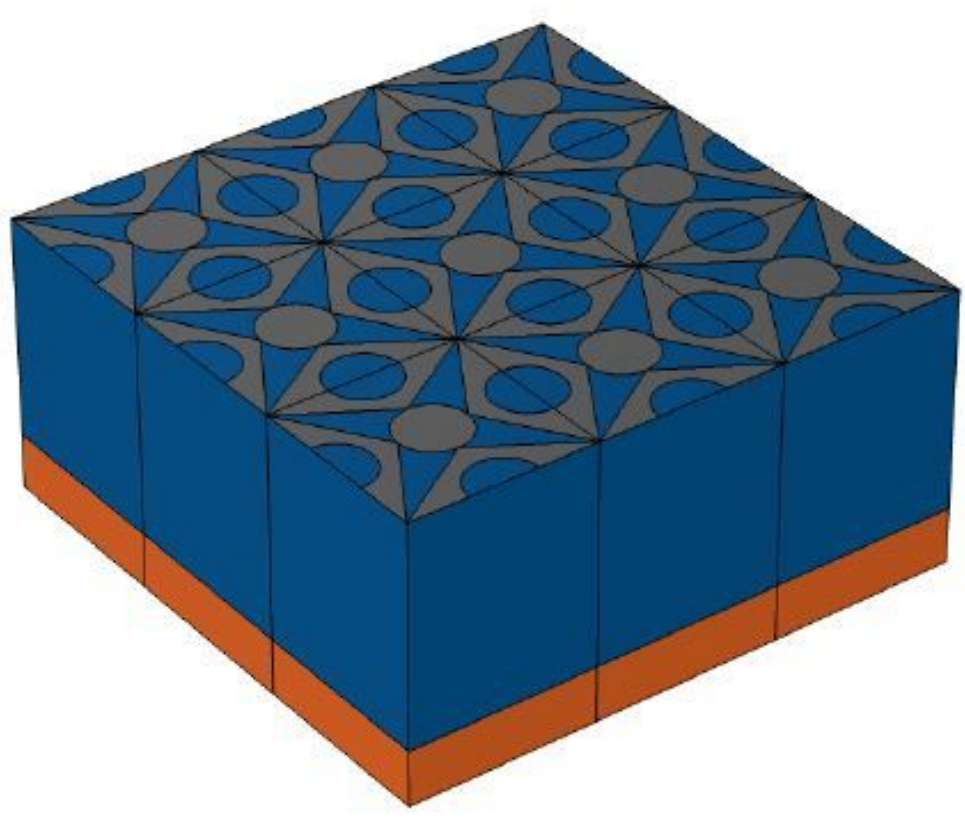

(a)

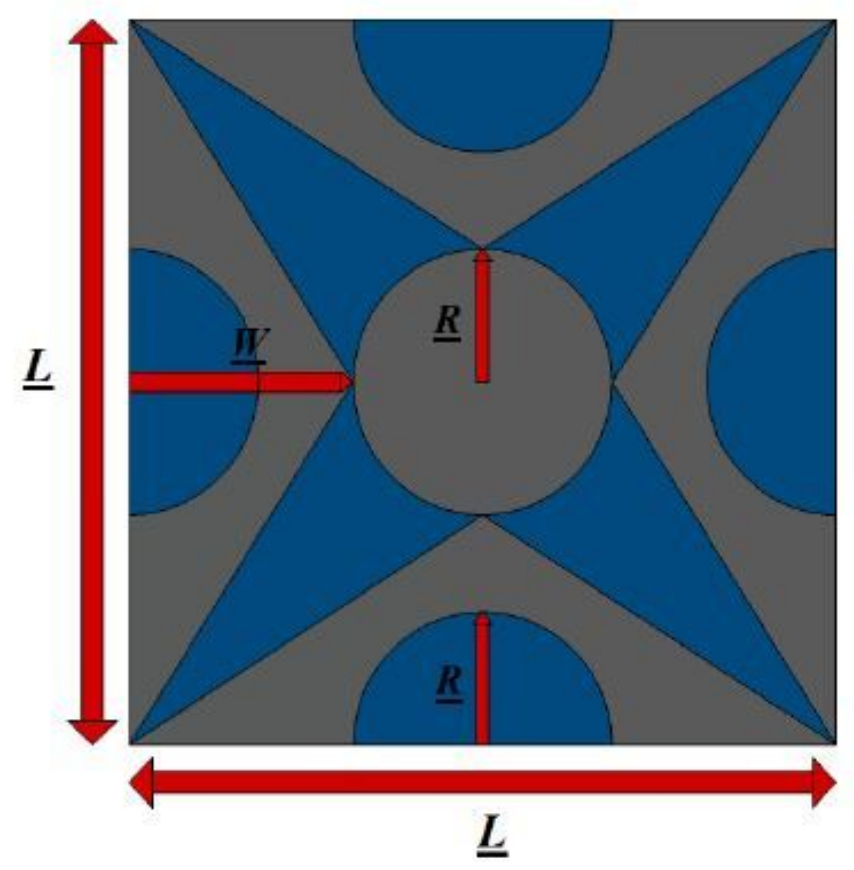

(b)

Figure 2

(a) Unit cells structure from three-dimensional view. (b) Dimensions of the absorber structure from a twodimensional view. (These figures were prepared using COMSOL Multiphysics software). 


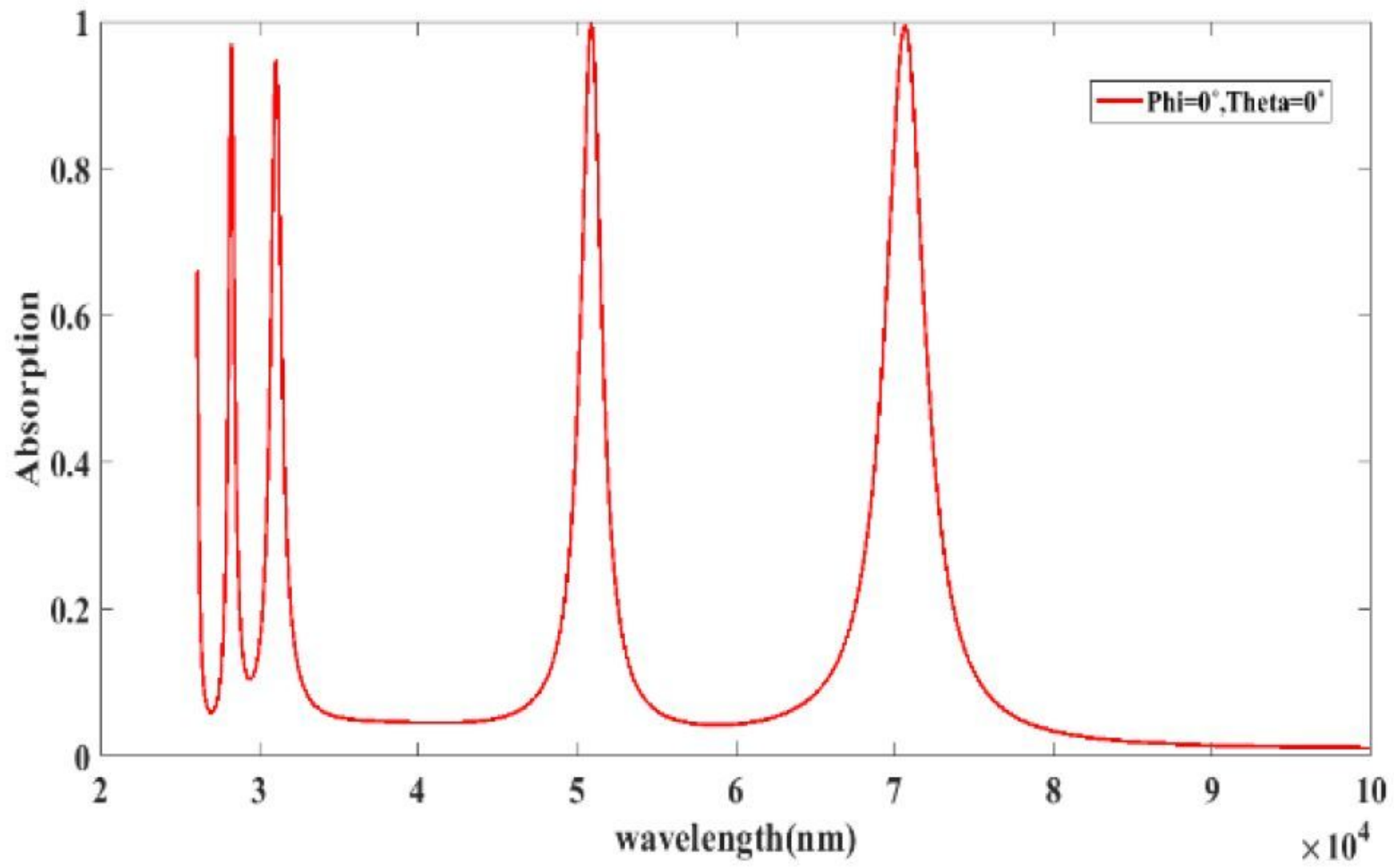

Figure 3

Absorption spectra of structure with $\mu \mathrm{c}=0.8 \mathrm{eV}$ and $\tau=1.6 \mathrm{ps}$. 


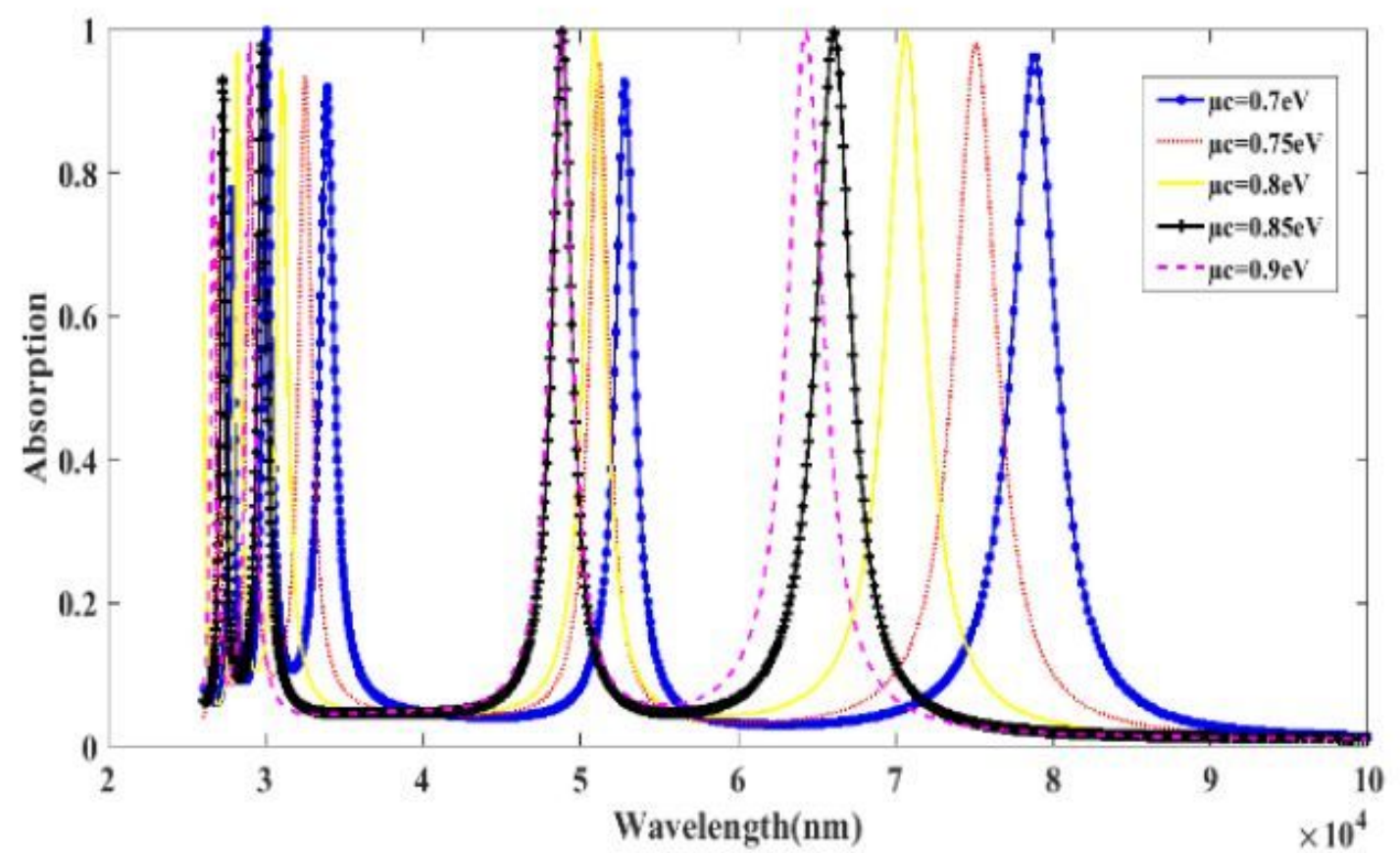

(a)

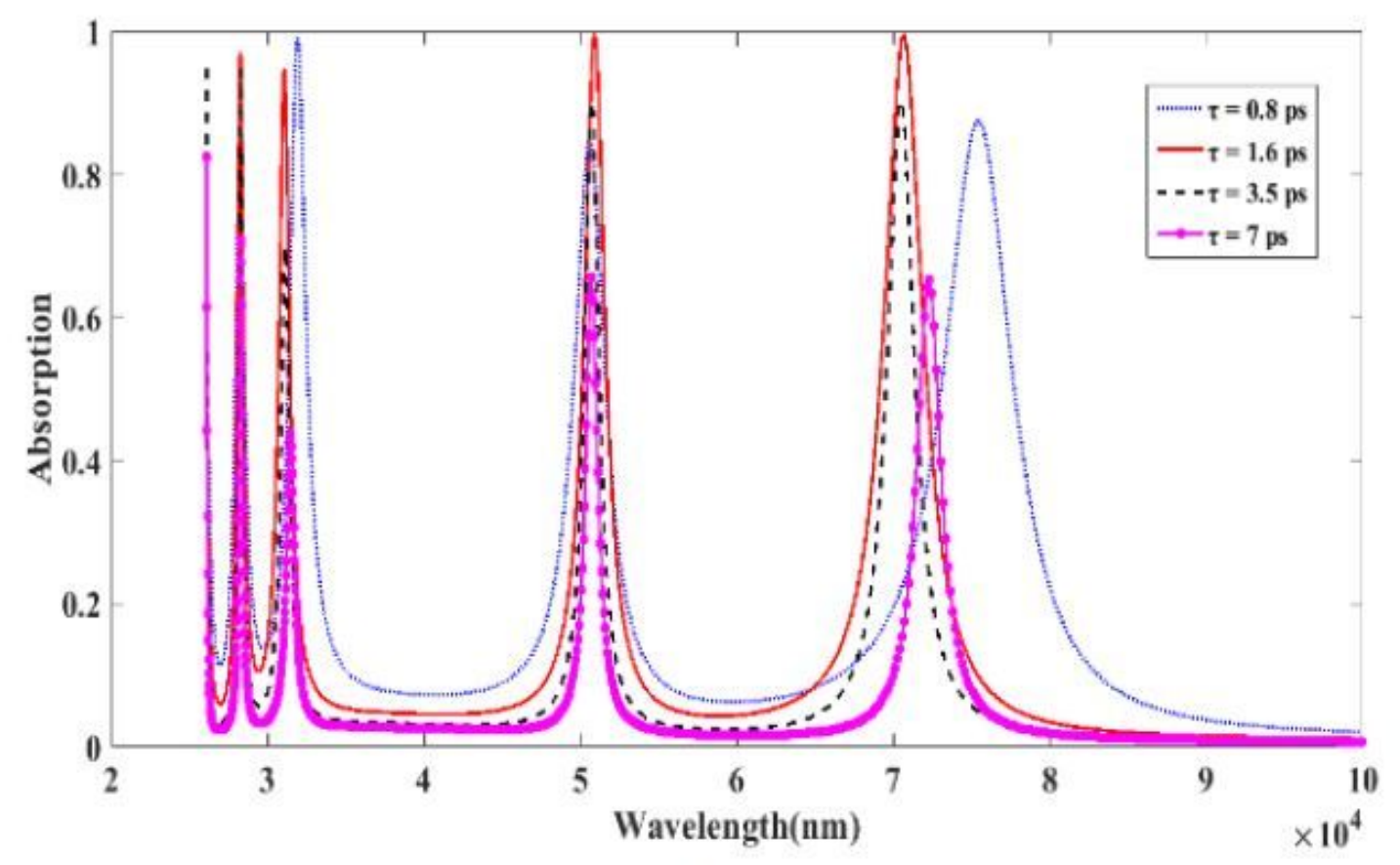

(b)

Figure 4

Absorption spectra of structure for (a) different Fermi level $\mu \mathrm{c}$ at $\tau=1.6$ ps and (b) different relaxation time $\tau$ at $\mu \mathrm{c}=0.8 \mathrm{eV}$. 


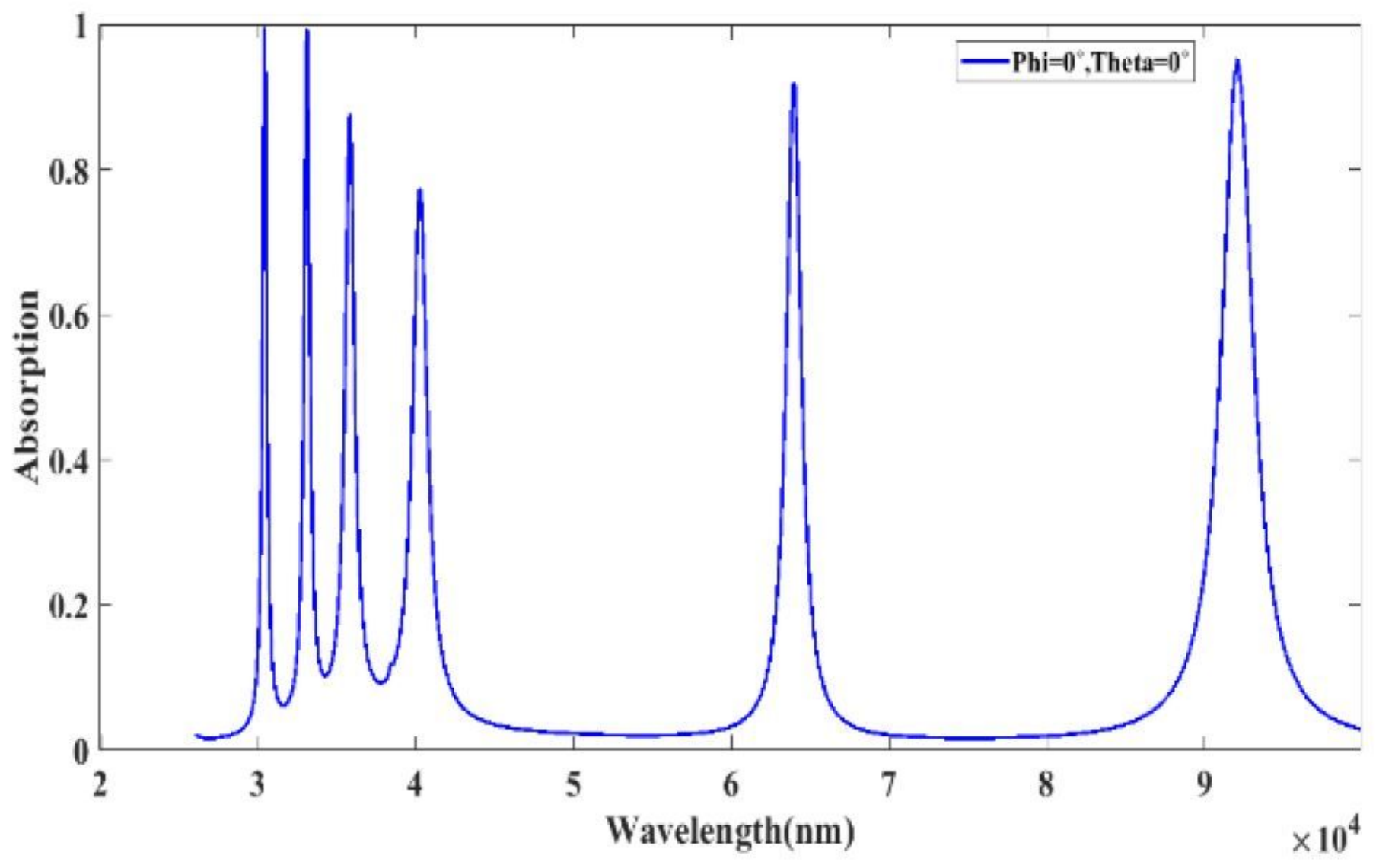

Figure 5

Increasing the number of absorption peaks by setting $\mu \mathrm{c}=0.5 \mathrm{eV}$ and $\tau=3 \mathrm{ps}$.

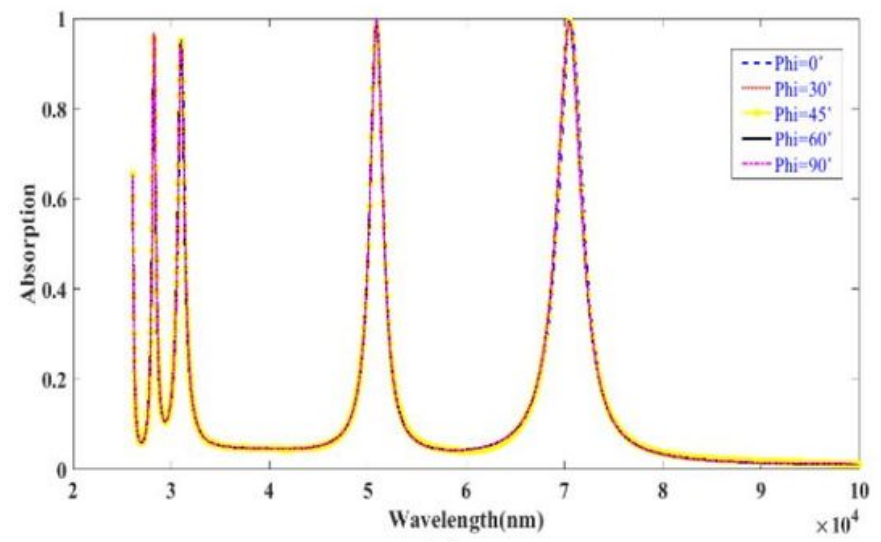

(a)

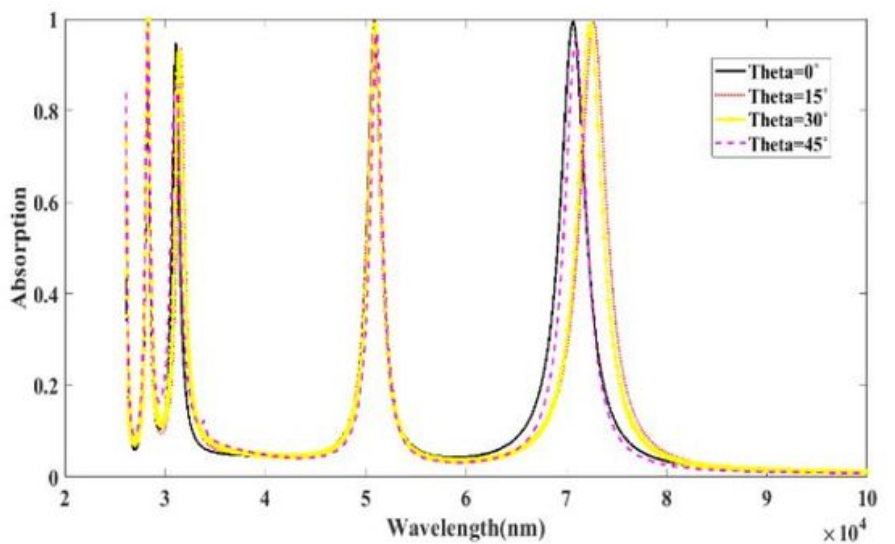

(b)

Figure 6

Absorption spectra of structure for (a) different polarization angles and (b) different incident angles. 
(a)

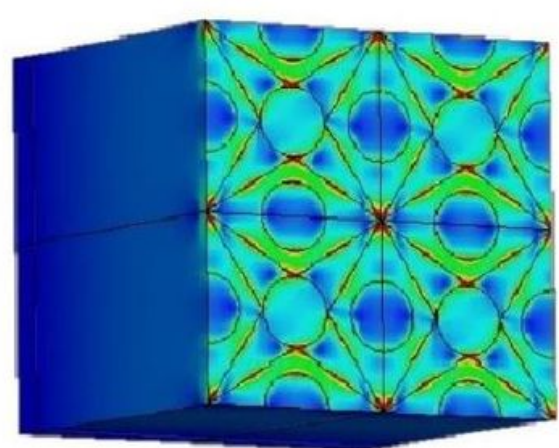

Max

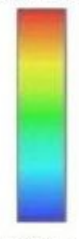

Min

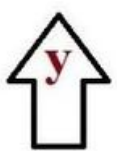

(b)
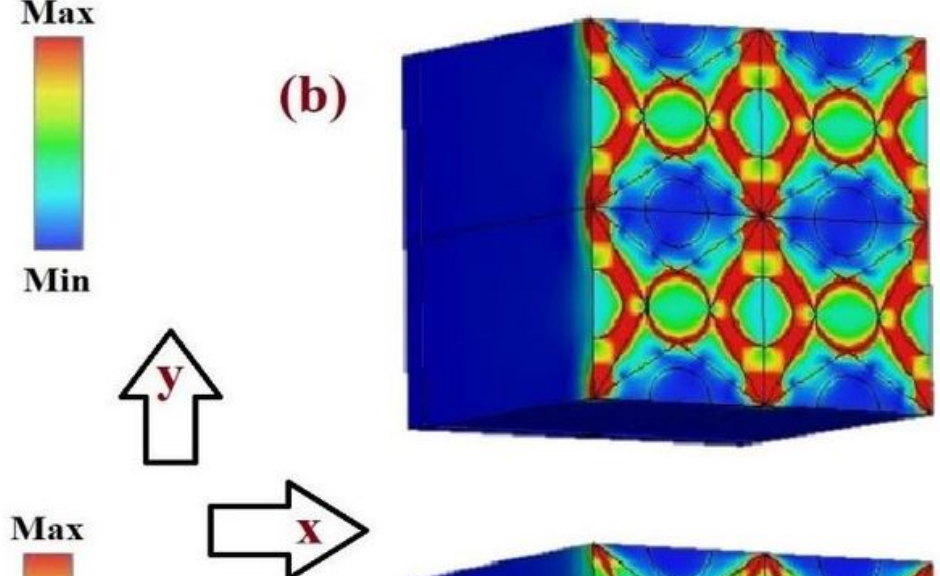

(d)

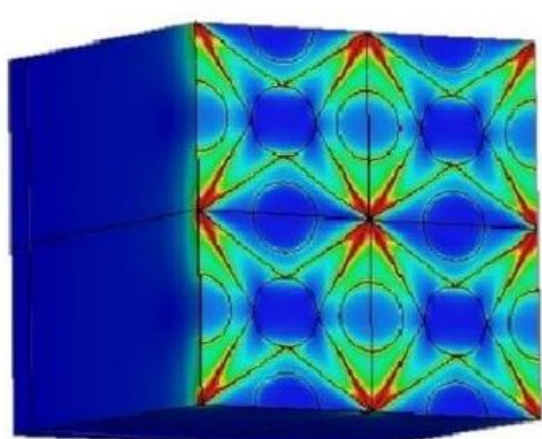

Max

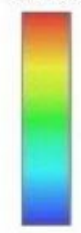

Min

Max

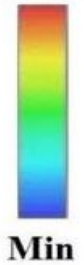

Figure 7

Electric field [real (Ez)] distributions in the normal incidence of TE waves at wavelengths of (a) $\lambda=$ $28239.7 \mathrm{~nm}$ (b) $\lambda=31048.9 \mathrm{~nm}$ (c) $\lambda=50898.6 \mathrm{~nm}$ (d) $\lambda=70689.1 \mathrm{~nm}$ 